\title{
Lactancia materna y cáncer de mama: un estudio caso-control en pacientes del Hospital Nacional Arzobispo Loayza, Lima-Perú *
}

\author{
Breastfeeding and breast cancer: a case-control study in patients at Arzobispo Loayza \\ National Hospital. Lima - Peru.
}

\author{
José Rojas Camayo ${ }^{1}$ \\ ${ }^{1}$ Médico-Cirujano. Facultad de Medicina de San Fernando, Universidad Nacional Mayor de San Marcos (UNMSM). Lima, Perú. \\ * Trabajo presentado como Tesis para optar el Título Profesional de Médico Cirujano en la Facultad de Medicina-UNMSM.
}

\section{Resumen}

Introducción: El cáncer de mama es un problema de salud pública a nivel mundial con un permanente incremento en su incidencia. La lactancia materna ha mostrado ser un factor protector del cáncer de mama en diferentes estudios; sin embargo, ha sido poco evaluado en poblaciones latinoamericanas. Objetivo: Evaluar el efecto de la lactancia materna sobre el riesgo de desarrollar cáncer de mama en una población peruana. Diseño: Estudio de tipo caso-control. Lugar: Hospital Nacional Arzobispo Loayza, Lima - Perú, en los servicios de Oncología Médica y Radiología (sección de Mamografía). Participantes: Pacientes con cáncer de mama y mujeres con mamografías Birads 1 y 2. Intervenciones: Se recolectó información de forma prospectiva, desde julio del 2006 hasta octubre del 2007. Se entrevistó 103 pacientes con cáncer de mama como casos y a 208 pacientes con mamografías Birads 1 y 2 como controles. Se consignó datos de factores de riesgo y patrones de lactancia materna. Se utilizó la estadística descriptiva, así mismo análisis bivariado y de regresión logística. Principales medidas de resultados: Protección de la lactancia materna sobre el cáncer de mama. Resultados: La ausencia de lactancia materna estuvo asociada con un ligero incremento no significativo del riesgo, comparado con las pacientes que por lo menos alguna vez dieron de lactar (OR ajustado 1,99; IC $95 \% 0,23$ a 6,99). Excluyendo las nulíparas, las que dieron de lactar de un mes a más por cada hijo comparadas con las que dieron de lactar menos de un mes por hijo tuvieron una reducción del riesgo, no significativo (OR ajustado 0,28: IC 95\% 0,06 a 1,27). Sin embargo, si comparamos la duración de lactancia total de las mujeres que dieron de lactar de 3 meses a más comparadas con las que dieron menos de 3 meses o nunca, tuvieron una reducción significativa del riesgo de desarrollar cáncer de mama (OR ajustado 0,24; IC 95\% 0,06 a 0,92). Conclusiones: La lactancia materna, en las mujeres que tuvieron hijos, muestra protección sobre el cáncer de mama, sobre todo si el período de lactancia materna total fue de tres meses a más.

Palabras clave: Lactancia materna; cáncer de mama; factores de riesgo.

\section{Abstract}

Introduction: Breast cancer is a global public health problem with incidence increasing in the world. Breastfeeding has been shown to be a protective factor, but evaluation in Latin American populations has scant. Objective: To evaluate the effect of breastfeeding on breast cancer developing risk in a Peruvian population. Design: A case-control study. Setting: Departments of Medical Oncology and Radiology, section of Mammography, at Arzobispo Loayza National Hospital, Lima - Peru. Participants: Patients with breast cancer and women with Birads 1 and 2 mammograms. Interventions: Information was collected from July 2006 through October 2007 from 103 patients with breast cancer as cases and 208 women with Birads 1 and 2 mammograms as controls. Interviews included information on risk factors and patterns of breastfeeding. We used descriptive statistics, and bivariate and logistic regression analysis. Main outcome measures: Breastfeeding protection on breast cancer. Results: Absence of breastfeeding was associated with a non- significant risk increase compared with patients that ever lactated (adjusted OR 1,99; $95 \% \mathrm{Cl} 0,23-6,99$ ). Excluding nulliparous, women that breastfed one month or more per child had a non-significant reduced risk compared with women that breastfed less than a month per child (adjusted OR 0,28, 95\% Cl 0,06-1,27). However, women who breastfed 3 months or more had a significant reduction in breast cancer risk compared with those that never breastfed or breastfed less than 3 months (adjusted OR 0,24; 95\% Cl 0,06-0,92). Conclusions: Breastfeeding by parous women showed protection on breast cancer risk especially if breastfeeding period was for three month or more.

Key words: Breast feeding; breast neoplasms; risk factors.

\section{INTRODUCCIÓN}

El cáncer mamario es un problema de salud pública a nivel mundial. De acuerdo con las estimaciones, en el año 2002, hubo 1151298 casos de cáncer diagnosticados y más de 4,4 millones que vivían con cáncer mamario en todo el mundo ${ }^{(1)}$. La incidencia y la mortalidad varían considerablemente en diferentes regiones. En general, la incidencia es alta (más de 80 por 100000 ) en las regiones desarrolladas y baja (menos de 30 por 100000 ), aunque en incremento, en regiones en desarrollo. La mortalidad es mucho más baja (aproxima- damente 6 a 23 por 100000 ) en las regiones más desarrolladas ${ }^{(2,3)}$. En el Perú, tomando los datos de Lima Metropolitana en los años 1994 a 1997, en mujeres, el cáncer de mama ocupó el primer lugar, con una tasa de incidencia de 32,3 por $100000^{(4)}$. En el Instituto Nacional de Enfermedades Neoplásicas, en el año 2002, se trató 1025 pacientes con cáncer de mama, siendo el segundo luego de cáncer de cuello uterino, con 1402 pacientes ${ }^{(5)}$.

Entre los factores de riesgo tenemos la edad, doblándose el riesgo a dos veces por cada diez años hasta la menopausia, cuando la tasa se lentifica dramáticamente. Comparado con el cáncer de pulmón, la incidencia del cáncer mamario es mayor en edades más jóvenes ${ }^{(6)}$. La incidencia y la mortalidad del cáncer mamario varían entre las poblaciones alrededor del mundo. Las tasas son más altas en los países desarrollados y bajas en los países en desarrollo y en Japón, aunque en este último está en incremento ${ }^{(3)}$. En cuanto a la historia familiar de cáncer de mama, según un estudio que reanalizó los datos de 52 estudios epidemiológicos y que incluyeron 58209 pacientes con cáncer de mama y 101986 
pacientes control, concluyeron que 8 de cada 9 mujeres en quienes se desarrolla cáncer mamario, no tienen a la madre, una hermana o una hija afectada. Aunque las mujeres que tienen parientes de primer grado afectados con cáncer de mama tienen un riesgo incrementado de desarrollar la enfermedad, sin embargo la mayoría nunca desarrollará la enfermedad y la mayoría de quienes lo desarrollan se les diagnosticará a una edad más allá de los 50 años ${ }^{(7)}$. Las mujeres con hiperplasia epitelial severa atípica tienen un riesgo de cuatro a cinco veces de desarrollar cáncer de mama comparadas con las que no tienen algún cambio proliferativo en sus mamas ${ }^{(1,6)}$. Las mujeres con este cambio y con historia familiar de cáncer mamario (de primer grado) tienen un incremento de nueve veces el riesgo de desarrollar el cáncer. Las mujeres con quistes palpables, fibroadenomas complejos, papilomas ductales, adenosis esclerosante y una hiperplasia epitelial moderada o florida tienen un incremento ligero del riesgo de cáncer ( 1,5 a 3 veces), comparado con mujeres que no tienen estos cambios; pero, este incremento no es clínicamente importante (6). Estudios de los patrones mamográficos del parénquima mamario muestran que las mujeres con mayor radiodensidad tienen un riesgo incrementado comparado con las mujeres con mamas más radiolucentes (8). Las mujeres que han sido expuestas a radiación ionizante, particularmente durante la pubertad, tienen un incremento del riesgo, aún con dosis bajas de exposición (9). Uno de los pocos estudios ocupacionales encontraron una alta mortalidad entre los tecnólogos de radiología en los Estados Unidos, especialmente sobre quienes trabajaron 30 años o más y en quienes fueron contratados antes de 1940, cuando las dosis fueron probablemente más altas ${ }^{(10)}$. El uso de dietiletilbestrol durante el embarazo, actualmente ya no empleado, por mujeres expuestas en los años 1940 a 1960, mostró un incremento de $30 \%$ del riesgo de cáncer de mama ${ }^{(11)}$. En cuanto al tabaco, según un estudio grande tiene poco o ningún efecto en el desarrollo de cáncer de mama ${ }^{(12)}$. Según los estudios observacionales, el alcohol incrementa de forma linear el riesgo de cáncer de mama ${ }^{(13)}$; un estudio menciona que el riesgo relativo se incrementa en 7,1\% por cada $10 \mathrm{~g} /$ día de consumo de alcohol ${ }^{(12)}$. La talla muestra una ligera asociación con el riesgo de cáncer de mama, la talla promedio es generalmente mayor en poblaciones con mayor incidencia que en poblaciones con menor incidencia, unos 10 centímetros más de talla está asociado con un incremento del riesgo en alrededor de $10 \%{ }^{(14)}$. En cuanto al peso al nacer, se ha comunicado que existe riesgo incrementado de cáncer de mama en mujeres cuyo peso al nacer fue alto ${ }^{(15)}$. El índice de masa corporal (IMC) tiene una sustancial asociación con el cáncer mamario en mujeres premenopáusicas y posmenopáusicas, respectivamente ${ }^{(16)}$. En las mujeres posmenopáusicas, en general la obesidad es un importante predictor de cáncer mamario ${ }^{(14,17)}$. Esto es debido probablemente a la asociación entre el incremento del IMC y un aumento de los estrógenos, principalmente estradiol biodisponible ${ }^{(18)}$. Las mujeres que actualmente usan anticonceptivos orales combinados o que los han usado en los últimos 10 años tienen un ligero incremento del riesgo de cáncer mamario; no hay evidencia de un incremento del riesgo luego de 10 años del cese del uso de anticonceptivos, y los cánceres detectados son menos avanzados clínicamente comparado con las mujeres que nunca usaron anticonceptivos orales ${ }^{(19)}$. La terapia de reemplazo hormonal incrementa el riesgo de cáncer mamario, especialmente con el uso prolongado ${ }^{(20,21)}$.

Entre los factores de riesgo reproductivos y hormonales tenemos a la edad de la menarquía: cuanto más tarde inicie la menstruación tendrá menor riesgo de desarrollar cáncer de mama ${ }^{(22)}$. Se menciona que existe un riesgo relativo de 3 en las mujeres con menarquía antes de los 11 años y se menciona además un riesgo relativo de 2 para las mujeres que tienen menopausia luego de los 54 años de edad ${ }^{(1,6)}$. MacHahon fue pionero en estudiar la asociación entre edad al primer nacimiento y paridad, con el riesgo de cáncer de mama ${ }^{(23)}$. La maternidad parece tener un efecto dual en el riesgo de cáncer mamario; se incrementa levemente en el período inmediato luego del nacimiento, pero gradualmente disminuye y a largo plazo el efecto de los nacimientos es proteger contra el cáncer mamario ${ }^{(24)}$. Comparado con las mujeres nulíparas, las mujeres que tuvieron al menos un embarazo a término, en promedio, tienen una reducción de $25 \%$ del riesgo ${ }^{(25,26)}$.

En los estudios caso control realizados en diferentes países, sobre todo en los desarrollados, se ha encontrado una relación inversa entre mayor lactancia materna y menor riesgo de cáncer de mama ${ }^{(25,27-40)}$. En cuanto a estudios de cohortes, hay cinco en la literatura mundial; tres de ellos mencionan que hay reducción del riesgo, principalmente en mujeres premenopáusicas ${ }^{(41-43)}$.
Sin embargo, en dos estudios de cohortes no se encontró esta relación ${ }^{(44,45)}$. A pesar de que los resultados no son convincentes en algunos estudios, la tendencia es que la lactancia materna es un factor protector significativo contra el cáncer de mama, como nos muestra un reanálisis de los datos individuales de 47 estudios epidemiológicos en 30 países, que incluyeron 50302 mujeres con cáncer de mama y 96973 mujeres sin la enfermedad. Se menciona que, entre las mujeres que tuvieron al menos un niño, $71 \%$ de las pacientes con cáncer de mama dio de lactar por lo menos una vez, versus $79 \%$ en los controles. El promedio para las pacientes con cáncer era 9,8 meses de lactancia comparado con 15,6 meses en los controles. Asimismo, se informa que el riesgo relativo se reduce en $4,3 \%$ por cada 12 meses de lactancia, en adición a una disminución de 7\% por cada nacimiento. Esta disminución del riesgo relativo del cáncer mamario asociada con la lactancia materna no varió de acuerdo a edad, estado menopáusico, origen étnico, número de hijos, edad al primer hijo y entre países desarrollados y en desarrollo. Dicho estudio concluye que cuanto más extenso sea el tiempo de lactancia, hay mayor protección contra el cáncer de mama ${ }^{(46)}$. Sin embargo, gran parte de estos estudios fueron realizados en países desarrollados y los datos de países en desarrollo son escasos. Además, los patrones de lactancia materna varían, con una tendencia a disminuir en los países desarrollados. No existen trabajos previos en el Perú que evalúen la relación entre la lactancia materna y el riesgo de cáncer de mama. Por ello, el objetivo del presente estudio era determinar la asociación entre lactancia materna y el riesgo de cáncer de mama, en un grupo de mujeres peruanas.

\section{MÉTODOS}

Se realizó un estudio caso control, que incluyó como casos a 103 pacientes mujeres con diagnóstico de cáncer de mama, que acudieron al Servicio de Oncología Médica del Hospital Nacional Arzobispo Loayza para consulta externa o cursos de quimioterapia. En cuanto a los controles, fueron 208 pacientes que acudieron al Servicio de Radiología a realizarse mamografías; se entrevistó a quienes tenían como resultado Birads (Breast Imaging Report and Data System) 1 (normal) y 2 (típicamente benigno) ${ }^{(47)}$. Ambos grupos fueron entrevistados de forma prospectiva, en el período comprendido entre julio de 2006 y octubre de 2007. 
Tanto a las pacientes con diagnóstico de cáncer de mama como a los controles se les realizó una entrevista personalizada, por un personal previamente capacitado, donde se consignó datos de edad, grado de instrucción, nacidos vivos, abortos, lactancia materna total y exclusiva por cada hijo, edad a la que tuvo su primer hijo, edad de la menarquia, estado menopáusico, edad de la menopausia, historia familiar de primer grado y número de familiares afectados, uso y tiempo en años de anticonceptivos hormonales, uso y tiempo en años de terapia de reemplazo hormonal, exposición a radiación ionizante, consumo de tabaco, antecedentes de patología mamaria benigna, consumo de alcohol en gramos/día (que se halló calculando el consumo de cerveza, vino y licor, referido según su frecuencia y cantidad, siendo la cantidad de alcohol: 5\%, 14\% y $40 \%$ respectivamente, realizando luego cálculos aritméticos para convertirlos en gramos/día), peso usual en la mayor parte de la vida adulta, peso actual, talla en metros y edad al diagnóstico de cáncer (casos). El tipo histológico fue tomado del reporte anatomopatológico de la historia clínica. Y para los controles el Birads 1 ó 2 resultantes de la mamografía. En cuanto a los datos antropométricos, se pesó y talló a las pacientes al momento de la entrevista. Todos estos datos fueron consignados en una sola ficha en la entrevista estructurada.

Los datos recolectados fueron consignados en una base datos, en el programa estadístico SPSS 13.0, utilizando la estadística descriptiva para la edad, grado de instrucción, tipo histológico, Birads en frecuencias y porcentajes. Se procedió luego a obtener las medias con sus respectivas desviaciones estándar y se realizó la prueba t-student, con su respectivo valor $\mathrm{p}$ de las variables cuantitativas.

Posteriormente, para el análisis multivariado mediante regresión logística ${ }^{(48)}$, se estratificó los factores de riesgo en: menarquia $(\geq 11,<11)$; paridad $(+3,2,1$, $0)$; abortos $(0,1,2+)$; edad al primer hijo $(<20,20-30,>30)$; menopausia $(\leq 54,>54)$; historia familiar de primer grado (no, sí); uso de anticonceptivos orales (no, sí); uso de terapia de reemplazo (no, sí); antecedente de patología benigna (no, sí); índice de masa corporal $(<25, \geq 25)$; exposición a radiación (no, sí); consumo de alcohol (no, sí) y lactancia (sí, no). Se obtuvo los OR mediante análisis bivariado inicialmente; y, luego, mediante análisis multivariado se obtuvo OR ajustados a las variables mencionadas anteriormente.
Finalmente, se realizó análisis multivariado, excluyendo a las pacientes nulíparas, para la variable tiempo de lactancia total ( $\geq 3$ meses, $<3$ meses) y para la variable lactancia por hijo ( $\geq 1 \mathrm{mes},<1 \mathrm{mes})$. Para ambas variables se obtuvo inicialmente los OR con análisis bivariado y luego, mediante regresión logística, los OR ajustados a edad, menarquia, paridad, abortos, edad al primer hijo, menopausia, historia familiar de primer grado, uso de anticonceptivos, uso terapia de reemplazo hormonal, antecedente de patología benigna, índice de masa corporal, exposición a radiación ionizante, consumo de alcohol, y consumo de tabaco. Se consideró para todos los casos una diferencia estadísticamente significativa para valores de OR del intervalo de confianza (IC) que no incluyeran la unidad, para un nivel de significancia del 95\%.

\section{RESULTADOS}

Se entrevistó 103 pacientes con cáncer de mama, que representaron los casos, y 208 pacientes sin cáncer de mama, con mamografía Birads 1 o 2, siendo los grupos etáreos de < 50 y de 50 a 70, los de mayor frecuencia; en cuanto al grado de instrucción se observa que $8,9 \%$ de los casos y $15 \%$ de los controles tuvieron instrucción universitaria; se aprecia también que $52 \%$ de los casos fue diagnosticado antes de los 50 años; el tipo histológico más frecuente fue el ductal simple, con $61,2 \%$ (Tabla 1 ).

En cuando a las características generales de los casos y controles sobre los factores de riesgo relacionados con cáncer de mama (Tabla 2), se puede observar que el promedio de número de familiares de primer grado afectados, el consumo de alcohol y el peso durante la mayor parte de la vida adulta, mostraron diferencia estadísticamente significativa. De manera peculiar se encontró mayor tiempo en años de uso de terapia de reemplazo hormonal en los controles comparado con los casos, 2,72 vs 1,80 ( $p=0,004)$, que podría tener explicación debido a que las pacientes en terapia de reemplazo hormonal acuden con más frecuencia a controles mamográficos y eran parte de la población que fue control y que acudía al servicio de mamografía. En cuanto al promedio de meses totales de lactancia entre los casos y controles, se observa que fue algo mayor en los casos, 40,1 vs. 35,63 , pero esta diferencia no es significativa $(p=0,35)$. Sin embargo, paradójicamente en el promedio de meses de lactancia exclusiva se observa la misma diferencia, pero alcanza a ser significativa 15,51 vs. $11,83(p=0,04)$.

Al analizar los factores de riesgo de cáncer de mama, mediante análisis bivariado y multivariado con regresión logística (Tabla 3), la menarquia antes de los 11 años no estuvo asociada con un mayor riesgo, OR ajustado 0,35 (IC 95\% 0,06 a $1,95)$. Teniendo como grupo de comparación a las mujeres que tuvieron tres a más hijos vivos, se observa una tendencia de incremento del riesgo, sin ser significativo, con respecto a las que tuvieron un hijo y ningún hijo (nulíparas), OR ajustado 1,24 (IC 95\% 0,58 a 2,66) y 2,88 (IC 95\% 0,41 a 20,44), respectivamente. Excluyendo las nulíparas y teniendo como referencia las mujeres que tuvieron su primer hijo antes de los veinte años, las que tuvieron entre los veinte a treinta años tuvieron un riesgo significativo incrementado, OR ajustado 1,97 (IC 95\% 1,02 a 3,82) y quienes lo tuvieron luego de los 30 años, OR ajustado

Tabla 1. Características demográficas, tipo histológico y Birads, de los casos y controles.

\begin{tabular}{|c|c|c|c|c|}
\hline & $\begin{array}{c}\text { Casos } \\
(n=103)\end{array}$ & Porcentaje & $\begin{array}{c}\text { Controles } \\
(n=208)\end{array}$ & Porcentaje \\
\hline \multicolumn{5}{|l|}{ Edad* $^{*}$} \\
\hline Menor de 50 & 48 & 46,6 & 112 & 54,4 \\
\hline De 50 a 70 & 46 & 44,7 & 93 & 45,1 \\
\hline Mayor de 70 & 9 & 8,7 & 1 & 0,5 \\
\hline \multicolumn{5}{|l|}{ Grado de instrucción ${ }^{\dagger}$} \\
\hline Ninguna & 5 & 5,0 & 14 & 6,8 \\
\hline Primaria & 36 & 35,6 & 59 & 28,5 \\
\hline Secundaria & 43 & 42,6 & 90 & 43,5 \\
\hline Técnico & 8 & 7,9 & 13 & 6,3 \\
\hline Universitario & 9 & 8,9 & 31 & 15,0 \\
\hline \multicolumn{5}{|c|}{ Edad al diagnóstico, en años ${ }^{\ddagger}$} \\
\hline$\leq 40$ & 17 & 17 & - & - \\
\hline 40 a 50 & 35 & 35 & - & - \\
\hline 50 a 60 & 30 & 30 & - & - \\
\hline 60 a 70 & 12 & 12 & - & - \\
\hline$>70$ & 6 & 6 & - & - \\
\hline \multicolumn{5}{|l|}{ Tipo histológico§ } \\
\hline Ductal simple & 63 & 61,2 & - & - \\
\hline Ductal medular & 3 & 2,9 & - & - \\
\hline Ductal comedón & 7 & 6,8 & - & - \\
\hline Ductal mixto & 1 & 1,0 & - & - \\
\hline Ductal in situ & 2 & 1,9 & - & - \\
\hline Lobulillar infiltrante & 13 & 12,6 & - & - \\
\hline Otro & 9 & 8,7 & - & - \\
\hline \multicolumn{5}{|l|}{ Birads"l } \\
\hline 1 & - & - & 72 & 35,5 \\
\hline 2 & - & - & 131 & 64,5 \\
\hline
\end{tabular}

* Dos controles no tuvieron edad conocida.

† Dos casos y un control no tuvieron datos de grado de instrucción.

¥ Tres casos no tuvieron datos disponibles de edad al diagnóstico.

$\S$ Cinco casos no tuvieron anatomía patológica disponible.

" Cinco controles no tuvieron datos disponibles de BIRADS. 
Tabla 2. Características de los casos y controles sobre los factores de riesgo relacionados con el cáncer de mama.

\begin{tabular}{lrrrrrr}
\hline & $\begin{array}{c}\text { Casos } \\
\text { Media }\end{array}$ & SD & $\begin{array}{c}\text { Controles } \\
\text { Media }\end{array}$ & SD & $t$ & $p$ \\
\hline Edad en años & 52,7 & 11,3 & 50,5 & 8,4 & $-1,73$ & 0,09 \\
Número de nacidos vivos & 3,6 & 2,4 & 3,3 & 2,1 & $-1,13$ & 0,26 \\
Número de abortos & 1,3 & 0,6 & 1,5 & 0,7 & 1,75 & 0,08 \\
Lactancia total en meses & 40,1 & 41,5 & 35,6 & 35,4 & $-0,94$ & 0,35 \\
Lactancia exclusiva en meses & 15,5 & 16,0 & 11,8 & 12,0 & $-2,06$ & $0,04^{*}$ \\
Lactancia materna total, por cada nacido vivo & 11,2 & 9,9 & 11,1 & 8,5 & $-0,04$ & 0,97 \\
Edad de menarquía & 12,9 & 1,7 & 13,1 & 1,7 & 0,50 & 0,62 \\
Edad a la que tuvo su primer hijo & 23,0 & 5,9 & 22,0 & 5,4 & $-1,35$ & 0,18 \\
Edad de la última menstruación (menopáusicas) & 47,1 & 4,3 & 45,7 & 6,4 & 0,65 & 0,52 \\
Número de familiares de primer grado afectados & 1,4 & 0,5 & 1,0 & 0,0 & $-2,50$ & $0,04^{*}$ \\
Tiempo en años del uso de anticonceptivos & 2,8 & 3,6 & 2,9 & 3,9 & 0,11 & 0,91 \\
Uso de terapia de reemplazo hormonal en años & 1,8 & 1,9 & 2,7 & 3,4 & 3,93 & $0,004^{*}$ \\
Consumo de alcohol en gramos/día & 3,6 & 8,8 & 1,3 & 4,2 & $-2,19$ & $0,03^{*}$ \\
Peso durante mayor parte de su vida adulta & 63,5 & 11,4 & 57,3 & 8,1 & $-4,91$ & $0,000^{*}$ \\
Peso actual cuantificado con balanza & 64,6 & 13,7 & 62,5 & 9,2 & $-1,40$ & 0,16 \\
Estatura de la paciente en metros & 1,6 & 0,1 & 1,6 & 0,1 & $-1,39$ & 0,17 \\
\hline
\end{tabular}

* Estadisticamente significativo para un $p<0,05$. riesgo de cáncer de mama, mostrando que la lactancia materna disminuye el riesgo de cáncer de mama de forma significativa.

Analizando el promedio de meses de lactancia por hijo y subdividiéndolos comparativamente en dos grupos -en menos de un mes de lactancia por hijo y en un mes a más de lactancia por hijo-, obtenemos en el análisis bivariado una disminución del riesgo entre quienes lactaron de un mes a más por hijo, OR 0,24 (IC 95\% 0,07 a 0,81); sin embargo, en el análisis multivariado, ajustando a los demás factores de riesgo, se obtuvo un disminución del riesgo, sin llegar a ser significativo, $O R$ ajustado 0,28 (IC 95\% 0,06 a 1,27).
1,72 (IC 95\%, 0,55 a 5,42). La edad de la menopausia mayor de 54 años tuvo discreto incremento del riesgo, sin ser significativo, OR ajustado 1,91 (IC 95\% 0,27 a 12,72). Una historia de tener familiares de primer grado afectados tuvo un OR ajustado no significativo de 2,19 (IC 95\% 0,76 a 6,28). El uso de anticonceptivos hormonales, un OR ajustado no significativo de 1,68 (IC $95 \% 0,94$ a 3,03). Uso de terapia de reemplazo hormonal, OR ajustado significativo de 0,17 (IC 95\% 0,06 a 0,51). El tener un antecedente de patología benigna de mama tuvo un riesgo significativo incrementado, OR ajustado 4,54 (IC 95\% 2,27 a 9,1). Un índice de masa corporal $\geq 25$ tuvo un OR ajustado no significativo de 0,94 (IC 95\% 0,53 a 1,66). El antecedente de exposición a radiación, $\mathrm{OR}$ ajustado no significativo de 0,57 (IC 95\% 0,32 a 1,02). Consumo de alcohol, OR ajustado no significativo de 1,28 (IC 95\% 0,68 a 2,43). Consumo de tabaco, OR ajustado no significativo de 1,94 (IC 95\% 0,90 a 4,17). En cuanto a presencia o no de lactancia materna, sin tomar en cuenta la duración, se obtiene un incremento del riesgo, sin llegar a ser significativo, OR ajustado 1,27 (IC95\%, $0,23$ a 6,99$)$.

$\mathrm{Al}$ analizar con mayor detalle los efectos de la lactancia materna, se excluyó a las mujeres que nunca tuvieron nacidos vivos (nulíparas), lo que se muestra en la Tabla 4. Estratificando a las mujeres según el tiempo de lactancia total en menos de tres meses y de tres meses a más, se observa en el análisis bivariado que las mujeres que dieron de lactar un total de tres meses a más, tuvieron un $\mathrm{OR}$ de 0,35 (IC 95\% 0,13 a 0,96), que se mantuvo incluso con el análisis multivariado, con OR ajustado de 0,24 (IC 95\% 0,06 a 0,92 ), ajustado los otros factores de
Tabla 3. Factores de riesgo de cáncer de mama, con OR ajustados.

\begin{tabular}{|c|c|c|c|c|c|c|}
\hline Factor & Casos & Controles & OR & IC 95\% & $\mathrm{OR}^{*}$ & IC 95\% \\
\hline \multicolumn{7}{|l|}{ Menarquia $^{\dagger}$} \\
\hline$\geq 11$ & $99(98,0 \%)$ & $196(95,6 \%)$ & 1,00 & & 1,00 & \\
\hline$<11$ & $2(2,0 \%)$ & $9(4,4 \%)$ & 0,44 & $(0,09-2,08)$ & 0,35 & $(0,06-1,95)$ \\
\hline \multicolumn{7}{|l|}{ Paridad } \\
\hline $3+$ & $56(54,4 \%)$ & $115(55,3 \%)$ & 1,00 & & 1,00 & \\
\hline 2 & $16(15,5 \%)$ & $47(22,6 \%)$ & 0,70 & $(0,35-1,40)$ & 0,69 & $(0,33-1,46)$ \\
\hline 1 & $17(16,5 \%)$ & $31(14,4 \%)$ & 1,13 & $(0,54-2,32)$ & 1,24 & $(0,58-2,66)$ \\
\hline 0 & $14(13,6 \%)$ & $15(7,7 \%)$ & 1,92 & $(0,81-4,55)$ & 2,88 & $(0,41-20,44)$ \\
\hline \multicolumn{7}{|l|}{ Abortos } \\
\hline 0 & $51(49,5 \%)$ & $117(56,3 \%)$ & 1,00 & & 1,00 & \\
\hline 1 & $39(37,9 \%)$ & $55(26,4 \%)$ & 1,63 & $(0,93-2,85)$ & 1,58 & $(0,84-2,98)$ \\
\hline $2+$ & $13(12,6 \%)$ & $36(17,3 \%)$ & 0,83 & $(0,38-1,78)$ & 0,88 & $(0,39-1,98)$ \\
\hline \multicolumn{7}{|l|}{ Edad al primer hijo } \\
\hline$<20$ & $26(29,2 \%)$ & $81(42,0 \%)$ & 1,00 & & 1,00 & \\
\hline $20-30$ & $53(59,6 \%)$ & $94(48,7 \%)$ & 1,76 & $(0,97-3,18)$ & 1,97 & $(1,02-3,82)^{\S \S}$ \\
\hline$>30$ & $10(11,2 \%)$ & $18(9,3 \%)$ & 1,73 & $(0,65-4,59)$ & 1,72 & $(0,55-5,42)$ \\
\hline \multicolumn{7}{|l|}{ Menopausia§ } \\
\hline$\leq 54$ & $61(95,3 \%)$ & $137(97,9 \%)$ & 1,00 & & 1,00 & \\
\hline$>54$ & $3(4,7 \%)$ & $3(2,1 \%)$ & 2,25 & $(0,44-11,45)$ & 1,91 & $(0,27-12,72)$ \\
\hline \multicolumn{7}{|c|}{ Historia familiar de primer grado } \\
\hline No & $93(90,3 \%)$ & $197(94,7 \%)$ & 1,00 & & 1,00 & \\
\hline Sí & $10(9,7 \%)$ & $11(5,3 \%)$ & 1,93 & $(0,79-4,70)$ & 2,19 & $(0,76-6,28)$ \\
\hline \multicolumn{7}{|c|}{ Uso anticonceptivo hormonall } \\
\hline No & $64(62,7 \%)$ & $141(67,8 \%)$ & 1,00 & & 1,00 & \\
\hline Sí & $38(37,3 \%)$ & $67(32,2 \%)$ & 1,25 & $(0,76-2,05)$ & 1,68 & $(0,94-3,03)$ \\
\hline \multicolumn{7}{|c|}{ Uso de terapia de reemplazo" } \\
\hline No & $96(94,1 \%)$ & $169(82,0 \%)$ & 1,00 & & 1,00 & \\
\hline Sí & $6(5,9 \%)$ & $37(18,0 \%)$ & 0,29 & $(0,12-0,70)^{\S \S}$ & 0,17 & $(0,06-0,51)^{\S \S}$ \\
\hline \multicolumn{7}{|c|}{ Antecedente de patología benigna** } \\
\hline No & $70(68,6 \%)$ & $186(89,9 \%)$ & 1,00 & & 1,00 & \\
\hline Sí & $32(31,4 \%)$ & $21(10,1 \%)$ & 4,05 & $(2,19-7,49)^{\S \S}$ & 4,54 & $(2,27-9,1)^{\S \S}$ \\
\hline \multicolumn{7}{|c|}{ Índice de masa corporalt+ } \\
\hline$<25$ & $42(41,6 \%)$ & $84(41,4 \%)$ & 1,00 & & 1,00 & \\
\hline$\geq 25$ & $59(58,4 \%)$ & $119(58,6 \%)$ & 0,99 & $(0,61-1,61)$ & 0,94 & $(0,53-1,66)$ \\
\hline \multicolumn{7}{|c|}{ Exposición a radiación ${ }^{\ddagger \ddagger}$} \\
\hline No & $44(42,7 \%)$ & $72(35,6 \%)$ & 1,00 & & 1,00 & \\
\hline Sí & $59(57,3 \%)$ & $130(64,4 \%)$ & 0,74 & $(0,46-1,21)$ & 0,57 & $(0,32-1,02)$ \\
\hline \multicolumn{7}{|l|}{ Consumo de alcohol } \\
\hline No & $25(24,3 \%)$ & $64(30,8 \%)$ & 1,00 & & 1,00 & \\
\hline Sí & $78(75,7 \%)$ & $144(69,2 \%)$ & 1,39 & $(0,81-2,38)$ & 1,28 & $(0,68-2,43)$ \\
\hline \multicolumn{7}{|l|}{ Consumo de tabaco } \\
\hline No & $83(80,6 \%)$ & $183(88,0 \%)$ & 1,00 & & 1,00 & \\
\hline Sí & $20(19,4 \%)$ & $25(12,0 \%)$ & 1,76 & $(0,93-3,35)$ & 1,94 & $(0,90-4,17)$ \\
\hline \multicolumn{7}{|l|}{ Lactancia } \\
\hline Sí & $85(82,5 \%)$ & $188(90,4 \%)$ & 1,00 & & 1,00 & \\
\hline No & $18(17,5 \%)$ & $20(9,6 \%)$ & 1,99 & $(1,00-3,95)$ & 1,27 & $(0,23-6,99)$ \\
\hline
\end{tabular}

*Ajustado a menarquia, paridad, abortos, historia familiar, anticonceptivos, terapia reemplazo hormonal, antecedente patología benigna, IMC, exposición a radiación, alcohol, tabaco, lactancia. Para la variable 'edad al primer hijo', se añade a la regresión la misma variable; y para la variable 'menopausia', se añade además la misma variable más 'edad al primer hijo'. ${ }^{\dagger}$ Dos casos y tres controles no tuvieron información disponible. ${ }^{\ddagger}$ Se excluyó las nuliparas. ${ }^{\S}$ Se excluyó premenopáusicas. "Un caso no tiene datos de uso de anticonceptivos. "Un caso y dos controles perdidos. ${ }^{*}$ Un caso y un control tienen datos perdidos. ${ }^{\dagger+}$ En dos casos y cinco controles no se pudo obtener IMC, ${ }^{\ddagger}$ Seis controles con datos perdidos. ${ }^{\S \S}$ Estadísticamente significativo para un IC $95 \%$. 
Tabla 4. Duración de lactancia materna y lactancia promedio por hijo, excluyendo a las mujeres nulíparas.

\begin{tabular}{|c|c|c|c|c|c|c|}
\hline Patrón lactancia & Casos & Controles & $\mathrm{OR}^{*}$ & IC 95\% & $\mathrm{OR}^{\dagger}$ & IC 95\% \\
\hline \multicolumn{7}{|l|}{ Tiempo lactancia total } \\
\hline$<3$ meses & $11(12,2 \%)$ & $9(4,7 \%)$ & 1,00 & & 1,00 & \\
\hline$\geq 3$ meses & $79(87,8 \%)$ & $184(95,3 \%)$ & 0,35 & $(0,13-0,96)^{\ddagger}$ & 0,24 & $(0,06-0,92)^{\ddagger}$ \\
\hline \multicolumn{7}{|l|}{ Lactancia por hijo } \\
\hline$<1$ mes & $9(10,0 \%)$ & $5(2,6 \%)$ & 1,00 & & 1,00 & \\
\hline$\geq 1 \mathrm{mes}$ & $81(90,0 \%)$ & $188(97,4 \%)$ & 0,24 & $(0,07-0,1)^{\ddagger}$ & 0,28 & $(0,06-1,27)$ \\
\hline
\end{tabular}

* Odd Ratio con análisis bivariado.

${ }^{\dagger}$ Ajustada a edad, menarquia, paridad, abortos, edad al primer hijo, menopausia, historia familiar de primer grado, uso de anticonceptivos, uso terapia de reemplazo hormonal, antecedente de patología benigna, índice de masa corporal, exposición a radiación ionizante, consumo de alcohol, y consumo de tabaco.

‡Estadísticamente significativo para un IC 95\%.

\section{DISCUSIÓN}

En el presente estudio se encontró una notable reducción en el riesgo (OR ajustado 0,24; IC 95\% 0,06 a 0,92) de desarrollar cáncer de mama en las pacientes que tuvieron una historia de lactancia de tres meses a más, sugiriéndonos que a más tiempo de lactancia hay reducción del riesgo. Estos hallazgos son consistentes con algunos estudios realizados en países desarrollados (31-34,49-52). También se encontró un efecto protector en cuatro estudios realizados en China, de las ciudades de Shangai ${ }^{(53)}$, Beijing ${ }^{(54)}$, Tianjin ${ }^{(55)}$ y en Shandong ${ }^{(38)}$. Sin embargo, otros estudios no encontraron esta relación ${ }^{(44,45)}$. En Latinoamérica sólo existen dos estudios que la analizan de manera específica, uno de México, donde se obtuvo la lactancia como factor protector ${ }^{(34)}$, mientras el otro, de Brasil, no encontró esta asociación ${ }^{(56)}$. La tendencia es encontrar poca o ninguna protección en los países desarrollados. Asimismo, la incidencia de cáncer mamario es alta en zonas desarrolladas (más de 80 por 100 000) y baja en zonas menos desarrolladas (menos de 30 por 100 000), pero está aumentando en todas estas regiones. La tendencia desfavorable es debida al aumento de los factores de riesgo, que incluyen un menor número de hijos y menor período de lactancia ${ }^{(2)}$.

Los factores reproductivos están claramente relacionados con el riesgo de cáncer de mama, como podemos observar en los resultados de un estudio que incluyó un reanálisis de los datos de 47 estudios epidemiológicos ${ }^{(46)}$, donde se halló una paridad promedio de 2,2 vs. 2,6 de los casos y controles, respectivamente. Se puede notar que la paridad es mayor en los controles; sin embargo, comparada con nuestros resultados son menores, 3,6 vs. 3,3, respectivamente. La edad al primer niño, 25,5 vs. 23,6, comparada con la obtenida por nosotros es un año mayor en cada grupo, 23 vs 22 , que nos señala una edad más temprana en la que tuvieron su primer hijo, en nuestra población. En cuanto al tiempo de lactancia total, mientras que el promedio de todos los estudios incluidos muestran unos 9,8 meses para los casos y 15,6 para los controles, en nuestro estudio encontramos promedios de 40,1 y 35,6, respectivamente, con largos periodos de lactancia. Esto podría ser en parte explicación a nuestro incidencia de 32,3 por 100000 habitantes ${ }^{(4)}$, que es relativamente menor comparada a la encontrada en estudios realizados en Estados Unidos, Canadá, Holanda, Islandia, Dinamarca, Italia, Israel, Reino Unido, Suecia, Suiza, Australia, Alemania, Noruega, Irlanda y Finlandia, con incidencia mayor de 50 por 100000 habitantes ${ }^{(6)}$. Esta explicación tendría sustento en el reanálisis mencionado anteriormente donde señalan que la incidencia de cáncer mamario en los países desarrollados podría ser reducido en más de la mitad, de 6,23 a 2,7 por 100 mujeres a la edad de 70 años, si las mujeres tuvieran un número promedio de hijos y duración de lactancia total como en los países en desarrollo; la lactancia podría contribuir en casi dos tercios de esa reducción estimada y la corta duración de la lactancia en zonas desarrolladas contribuye a la alta incidencia en esos países ${ }^{(46)}$.

Diversas hipótesis se han planteado para explicar los mecanismos de protección de la lactancia; una de ellas nos menciona que la supresión de la ovulación que ocurre durante la lactancia puede reducir la exposición a las hormonas de la vida reproductiva, en especial del estrógeno ${ }^{(57)}$. En diversos estudios se ha encontrado que los niveles de estrógeno disminuyen durante la lactancia, aumentando luego de que lactancia finaliza ${ }^{(58-60)}$; inclusive, los valores de estrógeno en los fluidos mamarios disminuyen durante la lactancia ${ }^{(61)}$. Estos períodos de baja exposición a estrógenos durante la lactancia podría ser un mecanismo que explique la reducción del cáncer mamario, debido a que los estrógenos están estrechamente implicados en la carcinogénesis del cáncer mamario ${ }^{(62)}$. Además, la lactancia aumenta la proporción de células diferenciadas en el parénquima mamario. la cual le confiere protección contra los carcinógenos ${ }^{(63)}$. Otro mecanismo podría ser el hecho que la lactancia podría ayudar a reducir el peso pregestacional, aunque la disminución ha demostrado ser mínima y los resultados aún no son convincentes ${ }^{(64-66)}$. Entonces, si la lactancia redujera el peso ganado durante el embarazo, disminuiría tanto el IMC como el riesgo, debido a que IMC elevados se asocian con niveles incrementados de estrógenos, principalmente estradiol biodisponible, en mujeres posmenopáusicas (18). También, hay evidencia que durante la lactancia se expresa el factor transformante de crecimiento beta (TGF- $\beta$ ), que es un factor de crecimiento negativo regulado hormonalmente en las células mamarias ${ }^{(67-69)}$. Finalmente, se ha planteado la reducción de las concentraciones de organoclorinas tóxicas en las mamas, con el incremento de la duración de la lactancia. Sin embargo, en varios estudios no se ha encontrado relación entre las organoclorinas y el cáncer mamario ${ }^{(70-72)}$.

Como en muchos estudios caso-control puede existir el riesgo de sesgo de memoria, en nuestro estudio se realizó personalmente entrevistas estructuradas, para disminuir la posibilidad de sesgos.

Como observamos anteriormente, la lactancia materna está asociada inversamente con el cáncer de mama y en países como el nuestro, donde aún son prevalentes períodos amplios de lactancia comparados con los países desarrollados, sería en parte la explicación a nuestra incidencia relativamente baja ${ }^{(2)}$. Siendo la lactancia uno de los pocos factores modificables para el riesgo de cáncer mamario, sería un motivo más para incentivar la lactancia materna en nuestro país, no solo por los beneficios al niño, ampliamente conocidos ${ }^{(73)}$, sino también por los beneficios a la madre en la disminución del riesgo de desarrollar cáncer mamario. Según la legislación peruana, en la Ley $\mathrm{N}^{\circ} 26644$ Artículo 1 se menciona que es derecho de la trabajadora gestante gozar de 45 días de descanso prenatal y 45 días de descanso posnatal ${ }^{(74)}$. Se observa que la tasa total de fecundidad, según el Endes 2004-2006, es 2,6, pero va disminuyendo ${ }^{(75)}$. Podemos afirmar que, en promedio una mujer aún trabajando puede superar el periodo de lactancia de 3 meses que se tomó como punto de corte en el estudio y de esta manera reducir el riesgo de cáncer mamario. 
Se concluye del presente trabajo que la lactancia materna, en las mujeres que han tenido hijos, ejerce un efecto protector que nos muestra que las mujeres que han dado de lactar de 3 meses a más tienen una reducción del riesgo de desarrollar cáncer mamario. Se recomienda además realizar estudios, tomando como base el presente, con mayor población, y multicéntricos, para evaluar el efecto de la lactancia en otros sectores de la población.

\section{AGRADECIMIENTOS}

Al Dr. Carlos Galarza Manyari. Instituto de Investigaciones Clínicas - UNMSM, por ser asesor del presente trabajo. Al Dr. César Gutiérrez Villafuerte. Departamento de Medicina Preventiva UNMSM, por su valioso apoyo en el análisis estadístico. Al Dr. Rolig Aliaga Chávez Jefe del Servicio de Oncología Médica del Hospital Nacional Arzobispo Loayza, por su apoyo en el diseño del proyecto y en el apoyo logístico en el estudio. Al Dr. Percy Pacora Portella, Departamento de Gineco-Obstetricia, Facultad de Medicina - UNMSM. Hospital Madre-Niño San Bartolomé, por su valioso apoyo en el diseño del proyecto del estudio. Al Dr. José Giglio, Jefe del Servicio de Patología Mamaria, Hospital Nacional Edgardo Rebagliati Martins, y al Dr. Vicente Vargas, médico asistente del Servicio de Oncologia Ginecológica y Mama del Hospital Nacional Arzobispo Loayza, por su apreciación y sugerencias en el desarrollo de la entrevista estructurada. A la Srta. Carolina Gallegos Campos, del Servicio de Oncología Médica, y la Sra. Magaly Quiroz Samamé, de la Sección de Mamografía del Servicio de Radiología del Hospital Nacional Arzobispo Loayza, por la seria realización de las entrevistas. A la Srta. Giovanna Martínez Martínez, por su incondicional apoyo logístico. Y a mis padres, Víctor Rojas Vásquez y Carmen Amalia Camayo Montalván, por apoyarme en todas las etapas de mi vida.

\section{REFERENCIAS BIBLIOGRÁFICAS}

1. Veronesi U, Boyle P, Goldhirsch A, Orecchia R, Viale G. Breast cancer. Lancet. 2005;365(9472):1727-41.

2. Parkin DM, Fernandez LM. Use of statistics to assess the global burden of breast cancer. Breast J. 2006;12 Suppl 1:S70-80.

3. Key TJ, Verkasalo PK, Banks E. Epidemiology of breast cancer. Lancet Oncol. 2001;2(3):133-40.

4. Centro de Investigación en Cáncer Maes Heller. Instituto Nacional de Enfermedades Neoplásicas. Registro de Cáncer de Lima Metropolitana 19941997. Lima: INEN; 2004.

5. Instituto Nacional de Enfermedades Neoplásicas. Estadística: Perfil Epidemiológico. Lima: INEN; 2006. Disponible en: http://www.inen.sld.pe/ intranet/estadepidemiologicos.htm

6. McPherson K, Steel CM, Dixon JM. ABC of breast diseases. Breast cancer-epidemiology, risk factors, and genetics. BMJ. 2000;321(7261):624-8.
7. Familial breast cancer: collaborative reanalysis of individual data from 52 epidemiological studies including 58,209 women with breast cancer and 101,986 women without the disease. Lancet. 2001;358(9291):1389-99.

8. Boyd NF, Lockwood GA, Byng JW, Tritchler DL, Yaffe MJ. Mammographic densities and breast cancer risk. Cancer Epidemiol Biomarkers Prev. 1998;7(12):1133-44.

9. Modan B, Chetrit A, Alfandary E, Katz L. Increased risk of breast cancer after low-dose irradiation. Lancet. 1989;1(8639):629-31.

10.Doody MM, Mandel JS, Lubin JH, Boice JD, Jr. Mortality among United States radiologic technologists, 1926-90. Cancer Causes Control. 1998;9(1):67-75.

11. Malone KE. Diethylstilbestrol (DES) and breast cancer. Epidemiol Rev. 1993;15(1):108-9.

12. Hamajima N, Hirose K, Tajima K, et al. Alcohol, tobacco and breast cancer--collaborative reanalysis of individual data from 53 epidemiological studies, including 58,515 women with breast cancer and 95,067 women without the disease. Br J Cancer. 2002;87(11):1234-45.

13. Smith-Warner SA, Spiegelman D, Yaun SS, et al. Alcohol and breast cancer in women: a pooled analysis of cohort studies. JAMA. 1998;279(7):53540.

14. Hunter DJ, Willett WC. Diet, body size, and breast cancer. Epidemiol Rev. 1993;15(1):110-32.

15. Michels KB, Trichopoulos D, Robins JM, et al. Birthweight as a risk factor for breast cancer. Lancet. 1996;348(9041):1542-6.

16. van den Brandt PA, Spiegelman D, Yaun SS, et al. Pooled analysis of prospective cohort studies on height, weight, and breast cancer risk. Am J Epidemiol. 2000;152(6):514-27.

17. Lahmann PH, Hoffmann K, Allen N, et al. Body size and breast cancer risk: findings from the European Prospective Investigation into Cancer And Nutrition (EPIC). Int J Cancer. 2004;111(5):762-71.

18. Key TJ, Appleby PN, Reeves GK, et al. Body mass index, serum sex hormones, and breast cancer risk in postmenopausal women. J Natl Cancer Inst. 2003;95(16):1218-26.

19. Breast cancer and hormonal contraceptives: collaborative reanalysis of individual data on 53 297 women with breast cancer and 100239 women without breast cancer from 54 epidemiological studies. Collaborative Group on Hormonal Factors in Breast Cancer. Lancet. 1996;347(9017):171327.

20. Breast cancer and hormone replacement therapy: collaborative reanalysis of data from 51 epidemiological studies of 52,705 women with breast cancer and 108,411 women without breast cancer. Collaborative Group on Hormonal Factors in Breast Cancer. Lancet. 1997;350(9084):1047-59.

21. Beral V. Breast cancer and hormone-replacement therapy in the Million Women Study. Lancet. 2003;362(9382):419-27.

22. Kelsey JL, Gammon MD, John EM. Reproductive factors and breast cancer. Epidemiol Rev. 1993;15(1):36-47.
23. MacMahon B. General Motors cancer research prizewinners laureates lectures. Charles S. Mott Prize. Reproduction and cancer of the breast. Cancer. 1993;71(10):3185-8.

24.Beral V, Reeves G. Childbearing, oral contraceptive use, and breast cancer. Lancet. 1993;341(8852):1102.

25. Layde PM, Webster LA, Baughman AL, Wingo PA, Rubin GL, Ory HW. The independent associations of parity, age at first full term pregnancy, and duration of breastfeeding with the risk of breast cancer. Cancer and Steroid Hormone Study Group. J Clin Epidemiol. 1989;42(10):963-73.

26. Ewertz M, Duffy SW, Adami HO, et al. Age at first birth, parity and risk of breast cancer: a metaanalysis of 8 studies from the Nordic countries. Int J Cancer. 1990;46(4):597-603.

27. Byers T, Graham S, Rzepka T, Marshall J. Lactation and breast cancer. Evidence for a negative association in premenopausal women. Am J Epidemiol. 1985;121(5):664-74.

28. McTiernan A, Thomas DB. Evidence for a protective effect of lactation on risk of breast cancer in young women. Results from a case-control study. Am J Epidemiol. 1986;124(3):353-8.

29. Siskind V, Schofield F, Rice D, Bain C. Breast cancer and breastfeeding: results from an Australian case-control study. Am J Epidemiol. 1989;130(2):229-36.

30. Yoo KY, Tajima K, Kuroishi T, et al. Independent protective effect of lactation against breast cancer: a case-control study in Japan. Am J Epidemiol. 1992;135(7):726-33.

31. Yang CP, Weiss NS, Band PR, Gallagher RP, White E, Daling JR. History of lactation and breast cancer risk. Am J Epidemiol. 1993;138(12):1050-6.

32. Breast feeding and risk of breast cancer in young women. United Kingdom National Case-Control Study Group. BMJ. 1993;307(6895):17-20.

33. Newcomb PA, Storer BE, Longnecker MP, et al. Lactation and a reduced risk of premenopausal breast cancer. N Engl J Med. 1994;330(2):81-7.

34. Romieu I, Hernandez-Avila M, Lazcano E, Lopez $L$, Romero-Jaime R. Breast cancer and lactation history in Mexican women. Am J Epidemiol. 1996;143(6):543-52.

35. Enger SM, Ross RK, Henderson B, Bernstein L. Breastfeeding history, pregnancy experience and risk of breast cancer. Br J Cancer. 1997;76(1):118-23.

36. Enger SM, Ross RK, Paganini-Hill A, Bernstein L. Breastfeeding experience and breast cancer risk among postmenopausal women. Cancer Epidemiol Biomarkers Prev. 1998;7(5):365-9.

37. Chang-Claude J, Eby N, Kiechle M, Bastert G, Becher $\mathrm{H}$. Breastfeeding and breast cancer risk by age 50 among women in Germany. Cancer Causes Control. 2000;11(8):687-95.

38.Zheng T, Duan L, Liu Y, et al. Lactation reduces breast cancer risk in Shandong Province, China. Am J Epidemiol. 2000;152(12):1129-35.

39.Zheng T, Holford TR, Mayne ST, et al. Lactation and breast cancer risk: a case-control study in Connecticut. Br J Cancer. 2001;84(11):1472-6. 
40. Kuru B, Ozaslan C, Ozdemir P, Dinc S, Camlibel $M$, Alagol H. Risk factors for breast cancer in Turkish women with early pregnancies and longlasting lactation--a case-control study. Acta Oncol. 2002;41(6):556-61.

41. Tryggvadottir L, Tulinius H, Eyfjord JE, Sigurvinsson T. Breastfeeding and reduced risk of breast cancer in an Icelandic cohort study. Am J Epidemiol. 2001;154(1):37-42.

42. Lee SY, Kim MT, Kim SW, Song MS, Yoon SJ. Effect of lifetime lactation on breast cancer risk: a Korean women's cohort study. Int J Cancer. 2003;105(3):390-3.

43. Martin RM, Middleton N, Gunnell D, Owen CG, Smith GD. Breast-feeding and cancer: the Boyd Orr cohort and a systematic review with meta-analysis. J Natl Cancer. Inst 2005;97(19):1446-57.

44. London SJ, Colditz GA, Stampfer MJ, et al. Lactation and risk of breast cancer in a cohort of US women. Am J Epidemiol. 1990;132(1):17-26.

45. Michels KB, Willett WC, Rosner BA, et al. Prospective assessment of breastfeeding and breast cancer incidence among 89,887 women. Lancet. 1996;347(8999):431-6.

46. Breast cancer and breastfeeding: collaborative reanalysis of individual data from 47 epidemiological studies in 30 countries, including 50302 women with breast cancer and 96973 women without the disease. Lancet. 2002;360(9328):187-95.

47. Balleyguier C, Ayadi S, Van Nguyen K, Vanel D, Dromain C, Sigal R. BIRADS classification in mammography. Eur J Radiol. 2007;61(2):192-4.

48. McKnight B, Cook LS, Weiss NS. Logistic regression analysis for more than one characteristic of exposure. Am J Epidemiol. 1999;149(11):984-92.

49. Katsouyanni K, Lipworth L, Trichopoulou A, Samoli E, Stuver S, Trichopoulos D. A case-control study of lactation and cancer of the breast. Br J Cancer. 1996;73(6):814-8.

50. Freudenheim JL, Marshall JR, Vena JE, et al. Lactation history and breast cancer risk. Am J Epidemiol. 1997;146(11):932-8.

51. Newcomb PA, Egan KM, Titus-Ernstoff L, et al. Lactation in relation to postmenopausal breast cancer. Am J Epidemiol. 1999;150(2):174-82.

52. Furberg H, Newman B, Moorman P, Millikan R. Lactation and breast cancer risk. Int J Epidemiol. 1999;28(3):396-402.
53. Yuan JM, Yu MC, Ross RK, Gao YT, Henderson BE. Risk factors for breast cancer in Chinese women in Shanghai. Cancer Res. 1988;48(7):1949-53.

54. Tao SC, Yu MC, Ross RK, Xiu KW. Risk factors for breast cancer in Chinese women of Beijing. Int J Cancer. 1988;42(4):495-8.

55. Wang QS, Ross RK, Yu MC, Ning JP, Henderson BE, Kimm HT. A case-control study of breast cancer in Tianjin, China. Cancer Epidemiol Biomarkers Prev. 1992;1(6):435-9.

56. Tessaro S, Beria JU, Tomasi E, Victora CG. Breastfeeding and breast cancer: a case-control study in Southern Brazil. Cad Saude Publica. 2003;19(6):1593-601.

57. Henderson BE, Ross RK, Judd HL, Krailo MD, Pike MC. Do regular ovulatory cycles increase breast cancer risk? Cancer. 1985;56(5):1206-8.

58. Said S, Johansson ED, Gemzell C. Serum oestrogens and progesterone after normal delivery. J Obstet Gynaecol Br Commonw. 1973;80(6):542-5.

59 . Endocrinology of human pregnancy. Res Reprod. 1975;7(2):1.

60. Shaaban MM, Sayed GH, Ghaneimah SA. The recovery of ovarian function during breastfeeding. J Steroid Biochem. 1987;27(4-6):104352.

61. Petrakis NL, Wrensch MR, Ernster VL, et al. Influence of pregnancy and lactation on serum and breast fluid estrogen levels: implications for breast cancer risk. Int J Cancer. 1987;40(5):587-91.

62. Yager JD, Davidson NE. Estrogen carcinogenesis in breast cancer. N Engl J Med. 2006;354(3):27082.

63. Russo J, Russo IH. Toward a physiological approach to breast cancer prevention. Cancer Epidemiol Biomarkers Prev. 1994;3(4):353-64.

64. Janney CA, Zhang D, Sowers M. Lactation and weight retention. Am J Clin Nutr. 1997;66(5):111624.

65. Olson CM, Strawderman MS, Hinton PS, Pearson TA. Gestational weight gain and postpartum behaviors associated with weight change from early pregnancy to 1 y postpartum. Int J Obes Relat Metab Disord. 2003;27(1):117-27.

66. Sichieri R, Field AE, Rich-Edwards J, Willett WC. Prospective assessment of exclusive breastfeeding in relation to weight change in women. Int J Obes Relat Metab Disord. 2003;27(7):815-20.
67. Knabbe C, Lippman ME, Wakefield LM, et al. Evidence that transforming growth factor-beta is a hormonally regulated negative growth factor in human breast cancer cells. Cell. 1987;48(3):41728.

68. Arteaga CL, Coffey RJ, Jr., Dugger TC, McCutchen CM, Moses HL, Lyons RM. Growth stimulation of human breast cancer cells with anti-transforming growth factor beta antibodies: evidence for negative autocrine regulation by transforming growth factor beta. Cell Growth Differ. 1990;1(8):367-74.

69. Burdette JE, Woodruff TK. Activin and estrogen crosstalk regulates transcription in human breast cancer cells. Endocr Relat Cancer. 2007;14(3):67989.

70.Wolff MS, Berkowitz GS, Brower S, et al. Organochlorine exposures and breast cancer risk in New York City women. Environ Res. 2000;84(2):151-61.

71.Laden F, Collman G, Iwamoto K, et al. 1,1Dichloro-2,2-bis(p-chlorophenyl)ethylene and polychlorinated biphenyls and breast cancer: combined analysis of five U.S. studies. J Natl Cancer Inst. 2001;93(10):768-76.

72. Gatto NM, Longnecker MP, Press MF, SullivanHalley J, McKean-Cowdin R, Bernstein L. Serum organochlorines and breast cancer: a case-control study among African-American women. Cancer Causes Control. 2007;18(1):29-39.

73. Newton ER. Breastmilk: the gold standard. Clin Obstet Gynecol. 2004;47(3):632-42.

74. Protección de la maternidad. El Peruano 27-061996; Sect. 140601-2.

75. Instituto Nacional de Enfermedades Neoplásicas. Encuesta Demográfica y de Salud Familiar. ENDES Continua 2004-2006. Lima: INEN; 2007.

Manuscrito recibido el 11 de enero de 2008 y aceptado para publicación el 15 de febrero de 2008.

\section{Correspondencia:}

José Ernesto Rojas Camayo

Jr. Canadá 319. San Martín de Porres

Lima 31, Perú

Correo-e: joserojas18@hotmail.com

jrojas@demina.com.pe 